\title{
INTEGRAÇÃO DOS MÉTODOS DE SÍSMICA DE REFRAÇÃO DE ONDA S E ANÁLISE MULTICANAL DE ONDAS SUPERFICIAIS (MASW) EM BARRAGEM DE TERRA
}

Leonides Guireli Netto (IPT), Otávio Coaracy Brasil Gandolfo (IPT), Walter Malagutti Filho (UNESP), João Carlos Dourado (UNESP)

Copyright 2019, SBGf - Sociedade Brasileira de Geofísica

This paper was prepared for presentation during the $16^{\text {th }}$ International Congress of the Brazilian Geophysical Society held in Rio de Janeiro, Brazil, 19-22 August 2019.

Contents of this paper were reviewed by the Technical Committee of the $16^{\text {th }}$ International Congress of the Brazilian Geophysical Society and do not necessarily represent any position of the SBGf, its officers or members. Electronic reproduction or storage of any part of this paper for commercial purposes without the written consent of the Brazilian Geophysical Society is prohibited.

\begin{abstract}
We present a data interpretation of seismic surveys using the S-wave refraction seismic and the multichannel analysis of surface waves - MASW methods in a small homogeneous earth dam in the city of Cordeirópolis, in the State of São Paulo. The association of these methods is recommended since both analyze the distribution of the S-wave velocity in subsurface. The objectives were identify the depth of lithological contacts and possible problems of compaction of the material that constitute the body of the dam, in complement with methods (resistivity and self potential) which were previously used in the study area.
\end{abstract}

\section{Introdução}

As barragens são estruturas construídas com o intuito de represar um material, água na maioria das vezes, através da construção de um bloqueio do fluxo principal de um rio, criando-se um reservatório artificial de acumulação de água.

As primeiras estruturas eram bastante simples e foram construídas de maneira empírica, ou seja, sem o devido critério técnico adequado, utilizando, na maioria dos casos, materiais próximos ao local de construção. Atualmente, os reservatórios são divididos em três tipos em relação ao material empregado na sua construção: terra, concreto e enrocamento.

No Brasil, casos de rompimentos de barragens ganharam bastante destaque devido aos recentes desastres ocorridos em Minas Gerais, nas cidades de Mariana (2015) e Brumadinhas (2019), causando enorme impacto ambiental, econômico e social, com inúmeras perdas de vidas humanas. Estes fatos chamaram a atenção para a necessidade de estudos nessa área de segurança de barragens.

A utilização integrada de métodos geofísicos constitui uma boa prática nos trabalhos de investigação em barragens para que sejam alcançados melhores resultados, citando-se, por exemplo, o emprego conjunto do método do potencial espontâneo (classicamente usado em barragens), dos métodos elétricos e dos métodos sísmicos.
A análise dinâmica de diques e barragens exige o conhecimento de um parâmetro fundamental, o módulo de rigidez dinâmico $\left(G_{\text {máx }}\right)$, que por sua vez pode ser determinado pela velocidade de propagação da onda cisalhante $\left(V_{S}\right)$ e pela densidade do solo $(\rho)$, através da expressão $G_{\text {máx }}=\rho \cdot V s^{2}$ (PARK e KISHIDA, 2018).

Apesar de ser a minoria (aproximadamente 10 mil barragens em um total de $200 \mathrm{mil}$ ), os barramentos de médio e grande porte detém o maior aporte de recursos para pesquisas geotécnicas e elaboração de projetos técnicos. Portanto, torna-se necessário um estudo mais detalhado das barragens de pequeno porte, não só por serem mais comuns, mas também por serem mais fáceis de serem investigadas, fazendo com que o conhecimento adquirido possa ser utilizado também em barragens de maiores portes.

Os métodos geofísicos podem ser uma ferramenta com ótimo custo/benefício, tanto na parte de implantação e construção da barragem, como na fase de manutenção e acompanhamento dos níveis de segurança da obra no decorrer do tempo. Estes métodos, se trabalhados em conjunto, podem auxiliar na identificação de fluxos internos anômalos e/ou zonas de maior percolação e de fraqueza na estrutura do barramento, por exemplo.

$O$ presente trabalho apresenta um estudo de caso no qual foram utilizados os métodos sísmicos do MASW (Multichannel Analysis of Surface Waves) e de refração com onda $S$, na avaliação da integridade de uma barragem de terra de pequeno porte localizada na cidade de Cordeirópolis, interior do Estado de São Paulo, com o objetivo de se definir a profundidade dos contatos litológicos que compõem o corpo da barragem, assim como identificar o topo rochoso sob o qual a barragem foi construída, uma vez que estes dados eram inexistentes.

\section{Métodos sísmicos MASW e Refração de onda S}

Os métodos sísmicos utilizados neste trabalho fornecem, como resultado final, informações sobre a distribuição em subsuperfície da velocidade de propagação da onda $S$ ou onda de cisalhamento $\left(V_{S}\right)$. Assim sendo, o emprego conjunto de ambas as técnicas torna-se adequado para a elaboração de um modelo mais confiável e representativo do substrato investigado. Resultados integrados de MASW com refração de ondas S podem ser encontrados em Turesson (2007), Schumann e Prado (2009), Gandolfo (2011), Gandolfo e Silva (2013), Pasquet et al. (2015).

$\mathrm{Na}$ aquisição dos dados são utilizados os mesmos equipamentos: sismógrafo, geofones e fonte para geração de ondas sísmicas. O arranjo de campo utilizado é também muito semelhante. 
O método MASW baseia-se no caráter dispersivo das ondas Rayleigh ao se propagar em um meio heterogêneo e estratificado. Do registro de campo (sismograma) é gerada uma imagem de dispersão obtida de uma dupla transformada de Fourier, da qual pode ser extraída uma curva de dispersão. A inversão dos dados desta curva fornece um modelo 1D de camadas, contendo o parâmetro $V_{S}$ versus a profundidade, correspondente a um ponto atribuído ao centro do arranjo linear dos geofones. Maiores informações sobre o método MASW podem ser obtidas em Foti (2000) e Strobbia (2003).

$\mathrm{Na}$ sísmica de refração com onda $\mathrm{S}$ utiliza-se uma fonte que gera preferencialmente este tipo de onda (por exemplo, uma marreta impactando um dormente de madeira em duas faces opostas) e geofones apropriados para captá-las (geofones de componente horizontal).

As ondas $S$ que se propagam em subsuperfície sofrem refração nas interfaces de camadas que apresentem contrastes em suas propriedades elásticas. A análise dos tempos de chegada destas ondas, em função da distância, permite a obtenção da distribuição de $V_{S}$ em um modelo 2D da subsuperfície.

A onda $S$, em virtude de algumas de suas características, vem sendo cada vez mais utilizada na geotecnia. Devido ao fato de ser praticamente insensível à presença de fluídos no maciço terroso, a sua velocidade de propagação sofre influência apenas das condições da matriz sólida do material e não de sua saturação.

$\mathrm{Na}$ avaliação da integridade física de barragens, materiais pouco consolidados apresentam menores valores de $V_{\mathrm{S}}$, enquanto que materiais com menor grau de alteração e/ou maior rigidez, apresentam maiores valores de $V_{S}$ e, logo, maiores condições necessárias para a manutenção da estrutura do barramento.

\section{Levantamentos de campo}

$\mathrm{Na}$ barragem estudada, no município de Cordeirópolis$\mathrm{SP}$, ocorre um solo laterítico argiloso, de coloração marrom avermelhada à marrom amarelado, proveniente de rochas básicas da Formação Serra Geral. Foi possível, nos trabalhos de campo, observar a presença de blocos e matacões de basalto nos arredores do barramento.

Trabalhos anteriores utilizando métodos geofísicos nesta área foram realizados por Camarero (2016) eletrorresistividade e por Guirelli Netto (2017) - potencial espontâneo.

Os levantamentos sísmicos foram realizados em outubro de 2018. A aquisição dos dados utilizou o mesmo arranjo, tanto para a sísmica de refração com onda $\mathrm{S}$, como para - MASW, sendo ambos coletados com o sismógrafo Geode (Geometrics) de 24 canais. O método MASW utilizou geofones de $4,5 \mathrm{~Hz}$ de componente vertical, enquanto que na aquisição de dados de sísmica de refração utilizou-se geofones de $28 \mathrm{~Hz}$ de componente horizontal.

Em ambos os métodos foi utilizada uma marreta como fonte de ondas sísmicas e o espaçamento entre geofones foi de 2 metros. No método MASW foram empregados tiros com offsets iguais a 4, 8, 12 e 16 metros de distância do primeiro geofone. $\mathrm{Na}$ refração, foram efetuados cinco pontos de tiro internos ao arranjo, constituindo uma base sísmica.
Foi realizada uma única linha de aquisição de dados de 86 metros de comprimento, composta por duas bases sísmicas (Figura 1).

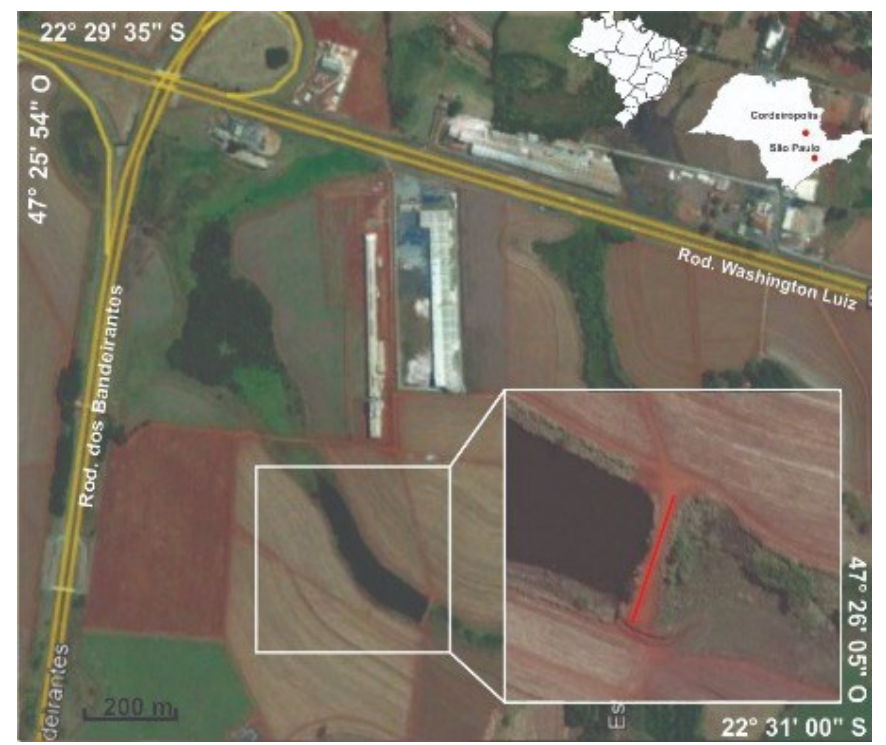

Figura 1. Localização da área de estudo e disposição da linha de aquisição de dados sísmicos, em vermelho. (modificado de GUIRELI NETTO, 2017).

A Figura 2 apresenta o levantamento de sísmica de refração com onda $S$ sendo realizado nos trabalhos de campo.

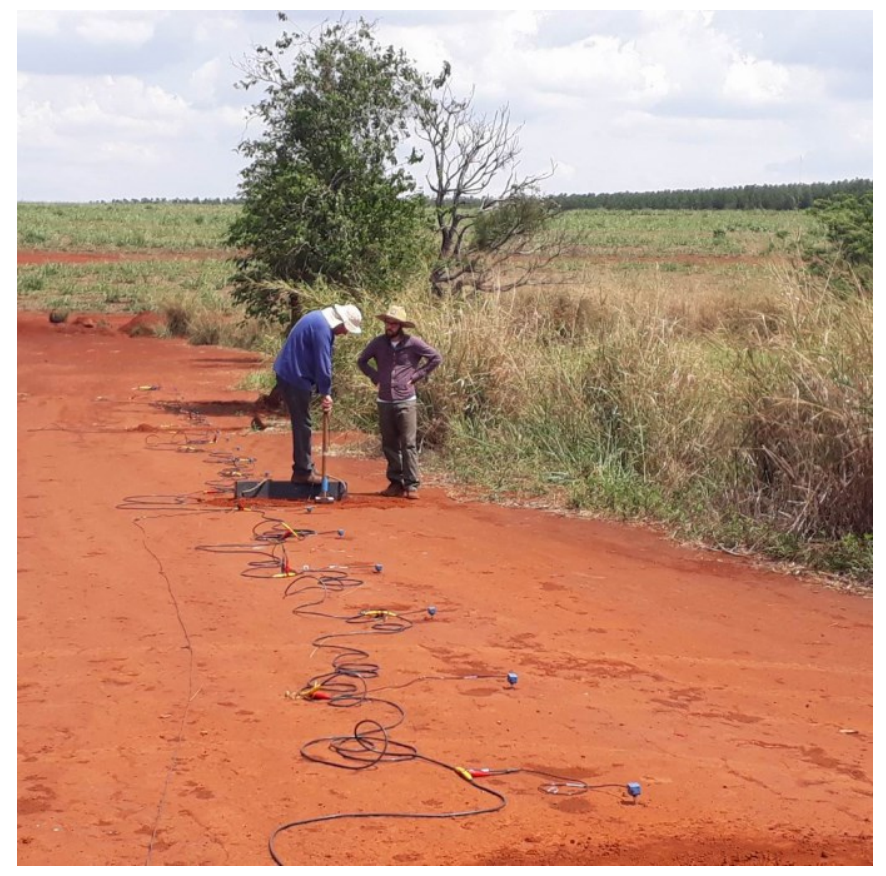

Figura 2. Levantamentos de sísmica de refração com onda S na barragem de Cordeirópolis. 


\section{Resultados}

A Figura 3 apresenta um sismograma da linha de aquisição, com o tiro realizado na posição igual a 31 metros (tiro central) da base 2. Deste registro, juntamente com os obtidos dos outros nove pontos de tiros realizados (bases 1 e 2), foram determinadas as primeiras quebras das ondas refratadas e construídos as respectivas curvas tempo $\mathrm{x}$ distância, que são mostradas na Figura 4.

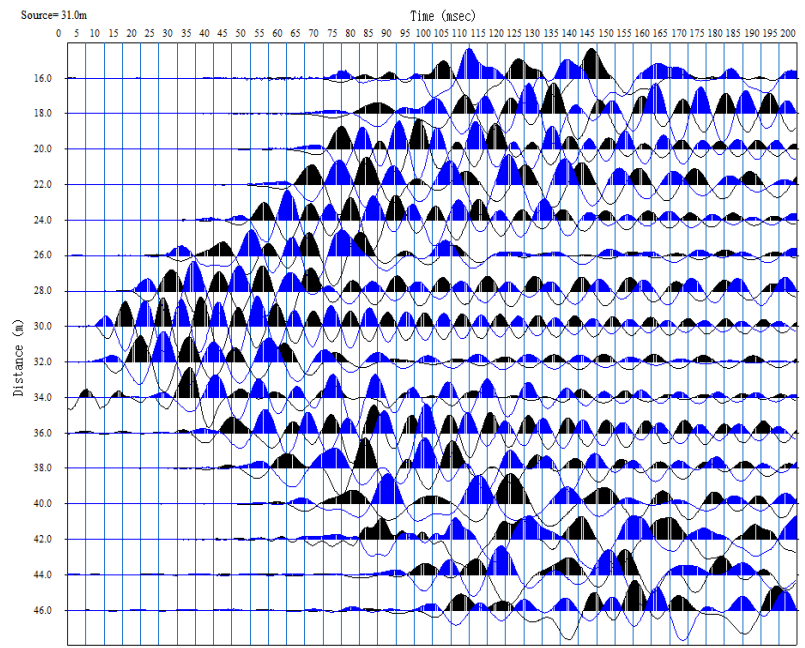

Figura 3. Sismograma com registro da onda $S$ obtido com o tiro na posição 31 metros da base 1 .

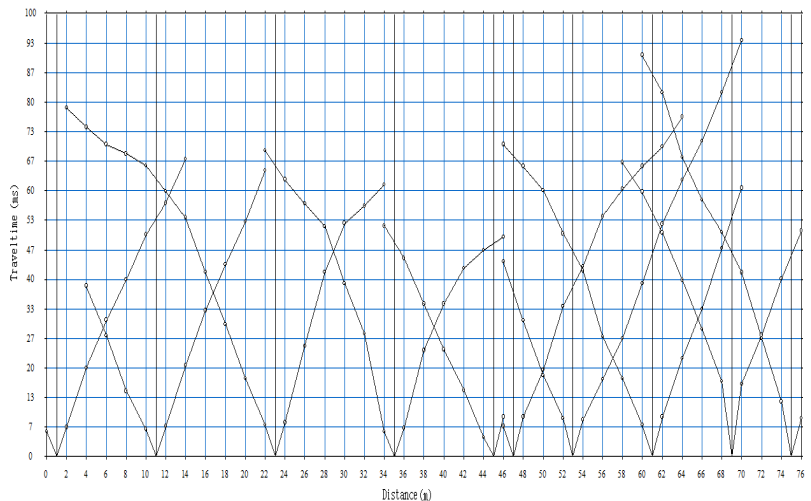

Figura 4. Curvas tempo $x$ distância correspondente aos dez tiros (cinco tiros por base) realizados na linha de aquisição sísmica de refração com onda S.

Pode ser verificada no gráfico da Figura 4 a ausência de informação em alguns dos 24 geofones do arranjo que constituíam uma base sísmica, pois não foi possível a determinação confiável dos tempos de chegada da onda S nestes geofones mais distantes dos pontos de tiros.

Por estar em uma área afastada do centro urbano e o fluxo de veículos serem praticamente inexistente, era de se esperar um baixo nível de ruído na barragem de Cordeirópolis - SP. Porém, a movimentação da água à montante gerava ruídos que prejudicaram a precisa determinação dos tempos de chegadas das ondas $S$ a partir de determinadas distâncias da fonte.

$\mathrm{Na}$ Figura 5 é apresentada a seção 2D modelada, obtida por inversão tomográfica utilizando o software Seis/mager.

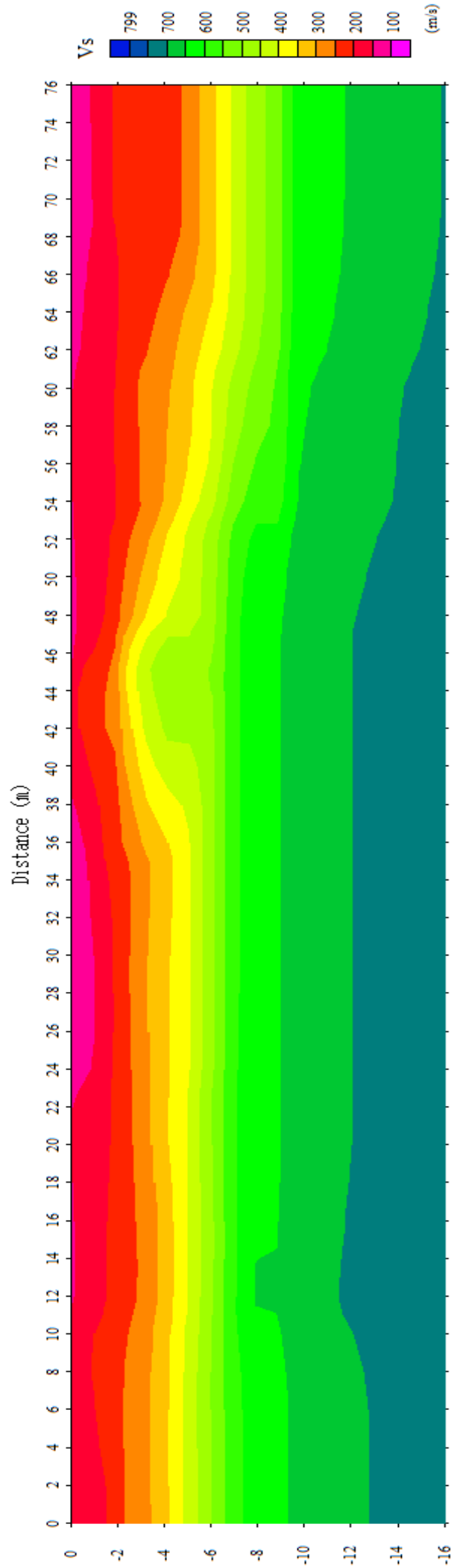

(III) чावन्व

Figura 5. Seção 2D modelada de refração de onda $S$ obtida por inversão tomográfica. 
Nas porções superiores são verificados valores de $V_{S}$ inferiores a $350 \mathrm{~m} / \mathrm{s}$, da superfície até a profundidade de aproximadamente 4 metros. Nesta porção foram identificadas duas regiões (em cor rosa na seção) nas distâncias entre 24-36 metros e 62-76 metros, provavelmente zonas de menor rigidez no corpo da barragem $\left(V_{S}\right.$ da ordem de $\left.100 \mathrm{~m} / \mathrm{s}\right)$, onde possivelmente o material não estaria muito bem compactado.

Pode também ser observado no modelo um aumento gradual de $V_{S}$ com a profundidade. Abaixo de 4 metros os valores de $V_{S}$ são superiores a $400 \mathrm{~m} / \mathrm{s}$

A Figura 6 apresenta a imagem de dispersão obtida do levantamento de MASW (offset igual a 16 metros), realizado na base 1 da linha sísmica. A respectiva curva de dispersão interpretada é mostrada na Figura 7.

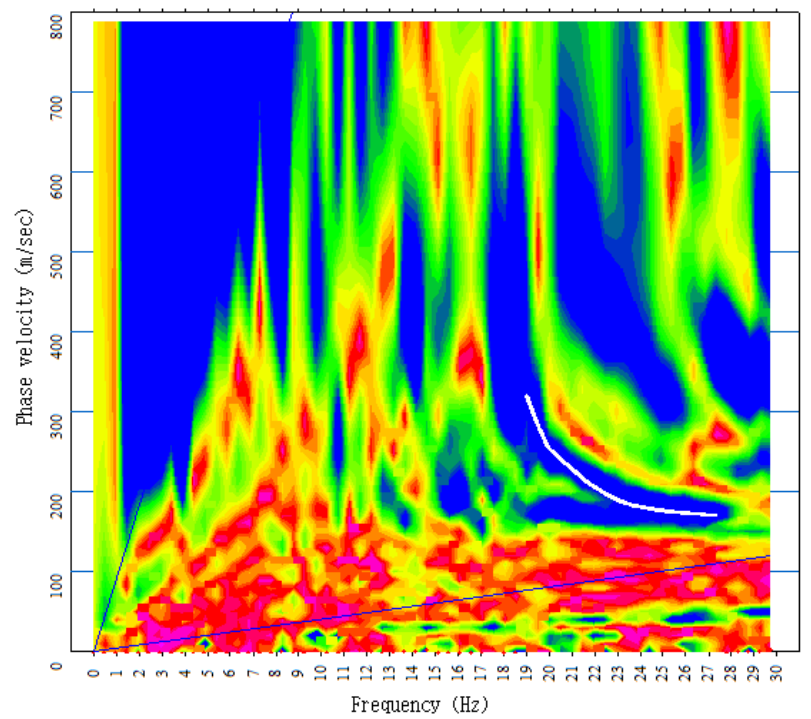

Figura 6. Imagem de dispersão obtida do levantamento MASW (base 1 da linha sísmica).

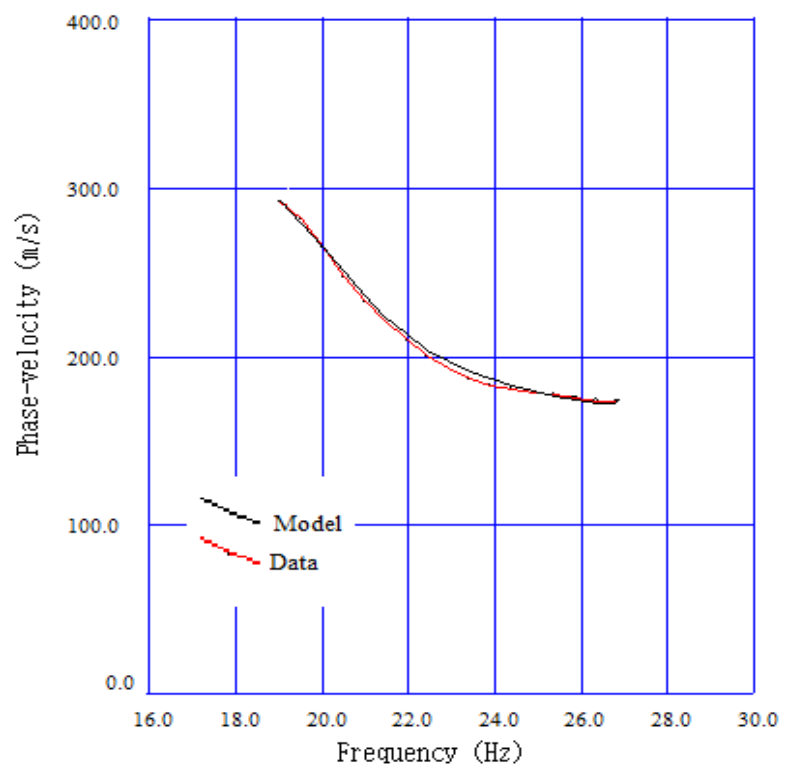

Figura 7. Curva de dispersão interpretada da imagem mostrada na Figura 6.
Pode haver uma ambiguidade na interpretação desta curva obtida da imagem de dispersão, pois os máximos de energia observados podem corresponder tanto ao modo fundamental como do primeiro modo superior. O software utilizado no processamento deste dado (Seis/mager/SW) permite apenas a inversão dos dados referentes ao modo fundamental de propagação.

Supondo, então, que a curva interpretada corresponde ao modo fundamental, foi efetuada a inversão dos dados que gerou o modelo $1 \mathrm{D}$ de $\mathrm{V}_{\mathrm{S}}$ versus a profundidade que é apresentado na Figura 8.

\section{S-welocity $(\pi / 1 / s)$}



Figura 8. Modelo de $\mathrm{V}_{\mathrm{S}}$ versus a profundidade, obtido por inversão de dados da curva de dispersão da Figura 7.

A análise dos dados de refração da onda $P$, assim como os resultados do levantamento de eletrorresistividade anteriormente realizado na área, indica a presença de duas interfaces no modelo, a aproximadamente 4 e 8 metros de profundidade. A camada situada a 8 metros provavelmente corresponde ao topo da rocha sã, não identificado pela refração com onda S.

A interface a aproximadamente 4 metros, já dentro da zona saturada do maciço, ficou muito bem caracterizada, tanto pela sísmica de refração com ondas $\mathrm{S}$ como a refração com onda $P$.

Na Figura 9 é apresentada a integração dos resultados dos modelos obtidos por refração de onda $S$ e MASW. Foi observada uma boa correlação dos dados. Próximo às regiões superficiais, as velocidades variam entre $150 \mathrm{e}$ $180 \mathrm{~m} / \mathrm{s}$, atingindo valores superiores a $350 \mathrm{~m} / \mathrm{s}$ abaixo da profundidade de 4 metros. 


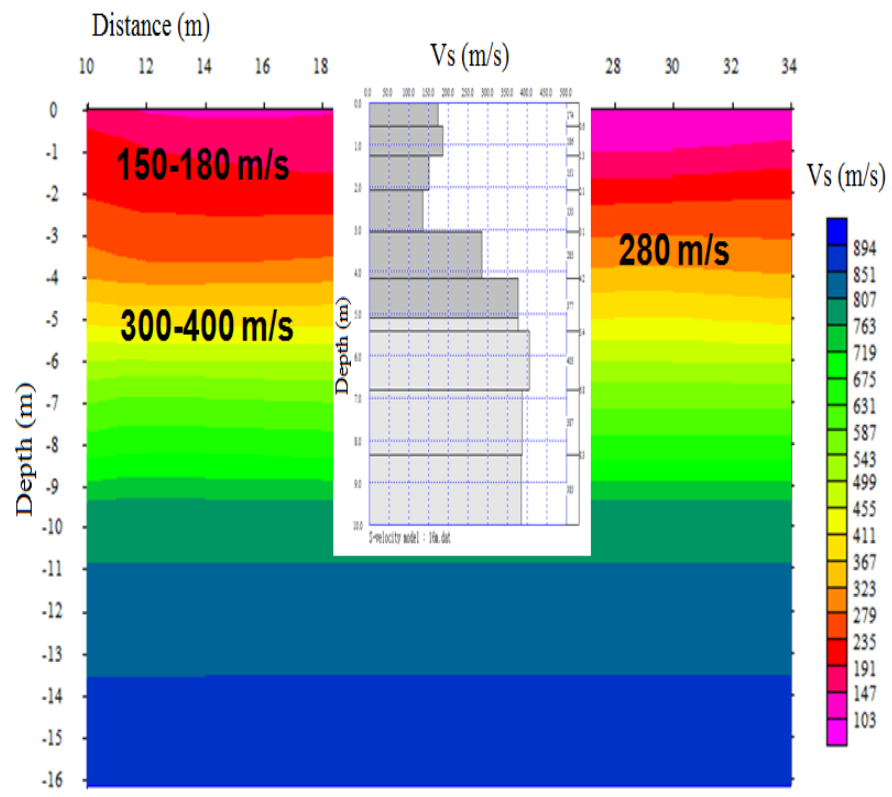

Figura 9. Integração dos modelos obtidos por refração de onda $S$ e MASW na barragem de Cordeirópolis (base 1 da linha sísmica).

\section{Conclusões}

Os recentes rompimentos de barragens no Brasil deixaram evidente a necessidade de investimentos em técnicas que auxiliem na administração dos níveis de segurança das barragens, sejam elas de contenção de rejeitos ou de água.

Os métodos geofísicos podem auxiliar no melhor domínio do comportamento da barragem e, caso sejam trabalhados em conjunto com equipamentos de investigações geotécnicas, agregam bastante no acompanhamento de situações que podem ocorrer no corpo do barramento ao longo do tempo.

O presente trabalho teve como objetivo determinar a profundidade de contatos litológicos no corpo da barragem, delimitar a base do topo rochoso e possíveis zonas de má compactação do solo que compõem o barramento de terra, complementando os trabalhos com os métodos de eletrorresistividade e potencial espontâneo, anteriormente realizados.

Os objetivos do estudo foram alcançados, mesmo em situações adversas, visto que não se tinha conhecimento do projeto de construção da barragem, que facilitaria bastante a localização de estruturas, como vertedouros, e da base da barragem.

O presente projeto deixou claro que o método de sísmica de refração, utilizando ondas $S$, associado ao método multicanal de ondas superficiais - MASW, podem ser uma interessante ferramenta em barragens de solo, para identificação de problemas de compactação do solo na estrutura do barramento.

Os levantamentos sísmicos devem, portanto, ser agregados aos outros métodos geofísicos classicamente empregados na avaliação de barragens, como a eletrorresistividade e o potencial espontâneo, resultando em um trabalho mais detalhado de investigação não destrutiva em subsuperfície em barragens de terra.

\section{Agradecimentos}

Os autores agradecem a Fundação de Amparo à Pesquisa do Estado de São Paulo, que através do Processo 2016/18080-9 financiou essa pesquisa.

\section{Referências Bibliográficas}

CAMARERO, P.L. 2016. Análise de integridade física de barragens de terra a partir da integração do método geofísico da eletrorresistividade com ensaios geotécnicos. Dissertação de Mestrado, IGCE-UNESP, $97 p$.

FOTI, S. 2000. Multistation Methods for Geotechnical Characterization using Surface Waves. PhD Thesis, Politecnico di Torino, 229p.

GANDOLFO, O.C.B. 2011. Ensaios sísmicos (refração utilizando ondas $P$ e $S$ e ensaio com ondas superficiais) na caracterização geotécnica de um aterro. In: $12^{\circ}$ International Congress of The Brazilian Geophysical Society, 2011, Rio de Janeiro. Expanded Abstracts (CD-ROM).

GANDOLFO O.C.B.; SILVA, I.V. 2013. A obtenção de velocidades da onda $\mathbf{S}$ por diferentes métodos sísmicos. In: $13^{\circ}$ International Congress of The Brazilian Geophysical Society, 2013, Rio de Janeiro. Expanded Abstracts (CD-ROM).

GUIRELI NETTO, L. 2017. Análise de fluxo no interior de barragens de terra a partir do método do potencial espontâneo (SP). Trabalho de Conclusão de Curso IGCE-UNESP, 88p.

PARK, D; KISHIDA. 2018. Shear wave velocity profiles of fill dams. Soil Dynamics and Earthquake Engineering, v. 104 , p. $250-258$.

PASQUET, S. et al. 2015. Detecting different water table levels in a shallow aquifer with combined P-, surface and SH-wave surveys: Insights from VP/VS or Poisson's ratios. Journal of Applied Geophysics, v. 113, p. 38-50.

SCHUMANN, F.B.; PRADO, R.L. 2009. O uso integrado dos métodos de sísmica de refração e de ondas superficiais visando à caracterização da subsuperfície rasa. In: $11^{\circ}$ International Congress of The Brazilian Geophysical Society, 2009, Salvador. Expanded Abstracts (CD-ROM).

STROBBIA, C. 2003. Surface Wave Method. Acquisition, processing and inversion. $\mathrm{PhD}$ Thesis, Politecnico di Torino, 317p.

TURESSON, A. 2007. A comparison of methods for the analysis of compressional, shear, and surface wave seismic data, and determination of the shear modulus. Journal of Applied Geophysics, v. 61, n. 2, p. 83-9. 